\title{
Transcriptome Analysis of Spermophilus lateralis and Spermophilus tridecemlineatus Liver Does Not Suggest the Presence of Spermophilus-Liver-Specific Reference Genes
}

\author{
Bryan M. H. Keng, ${ }^{1}$ Oliver Y. W. Chan, ${ }^{1}$ Sean S. J. Heng, ${ }^{1}$ and Maurice H. T. Ling ${ }^{2,3}$ \\ ${ }^{1}$ Raffles Institution, One Raffles Institution Lane, Singapore 575954 \\ ${ }^{2}$ Department of Zoology, The University of Melbourne, Genetics Lane, Parkville, VIC 3010, Australia \\ ${ }^{3}$ Department of Mathematics and Statistics, South Dakota State University, SD 57007, USA \\ Correspondence should be addressed to Maurice H. T. Ling; mauriceling@acm.org
}

Received 25 March 2013; Accepted 23 April 2013

Academic Editors: A. Bolshoy and D. Labudde

Copyright (C) 2013 Bryan M. H. Keng et al. This is an open access article distributed under the Creative Commons Attribution License, which permits unrestricted use, distribution, and reproduction in any medium, provided the original work is properly cited.

\begin{abstract}
The expressions of reference genes used in gene expression studies are assumed to be stable under most circumstances. However, studies had demonstrated that genes assumed to be stably expressed in a species are not necessarily stably expressed in other organisms. This study aims to evaluate the likelihood of genus-specific reference genes for liver using comparable microarray datasets from Spermophilus lateralis and Spermophilus tridecemlineatus. The coefficient of variance (CV) of each probe was calculated and there were 178 probes common between the lowest $10 \% \mathrm{CV}$ of both datasets $(n=1258)$. All 3 lists were analysed by NormFinder. Our results suggest that the most invariant probe for $S$. tridecemlineatus was $02 \mathrm{n} 12$, while that for $S$. lateralis was 24j21. However, our results showed that Probes $02 \mathrm{n} 12$ and $24 \mathrm{j} 21$ are ranked 8644 and 926 in terms of invariancy for S. lateralis and S. tridecemlineatus respectively. This suggests the lack of common liver-specific reference probes for both $S$. lateralis and $S$. tridecemlineatus. Given that S. lateralis and S. tridecemlineatus are closely related species and the datasets are comparable, our results do not support the presence of genus-specific reference genes.
\end{abstract}

\section{Introduction}

Gene expression analysis is examining the variations in gene expression by measuring DNA expression levels over time. These variations may be a result of many factors, such as environmental, developmental, and metabolic changes, or treatments. Quantitative real-time polymerase chain reaction (qRT-PCR) is one such used technique to quantify and analyse gene expressions $[1,2]$. However, qRT-PCR requires a stably expressed gene under a wide variety of conditions $[3,4]$, known as a reference gene, as a standard to produce accurate and reliable results on transcriptional differences of various genes of interest.

Candidate reference genes, which are commonly assumed to be invariant, can be identified using statistically based algorithms, such as geNorm [5], NormFinder [6], and BestKeeper [7], or descriptive statistics, such as regression [8].
Microarrays, which usually contain thousands of probes, present a good source of data for identifying reference genes [9]. Reference genes had been successfully identified from microarrays in a number of studies $[10,11]$.

However, several studies had refuted the possibility of universal reference genes [10-14] that can be used in every organ in every organism. This corroborates several studies demonstrating that genes commonly considered to be expressionally invariable may vary under different experimental conditions [15-17]. Some studies had verified the applicability of commonly used reference genes such as GAPDH (glyceraldehyde-3-phosphate dehydrogenase) [18] or $U B Q$ (ubiquinone) [18]. However, other studies had demonstrated that the expressions of GAPDH [19] and UBQ [20] vary in some conditions. Polr32 has been suggested to be stably expressed in mouse heart [21], but Mamo et al. [22] had shown that Polr32 is not stably expressed in mouse 
oocytes and embryos. GAPDH and PPIA are suitable reference genes for the human heart [23], but these genes were found to be unsuitable reference genes [24] for the human brain, dura mater, and meningiomas. These suggest that established reference genes for a particular organism may not be suitable for other organisms [25]. Using expressionally variable genes as reference genes will confound the results as it will be impossible to attribute the variation of the reference gene from the gene of interest. Given the lack of universal reference genes and the unlikelihood of organismspecific reference genes for multicellular organisms, the possibility of reference genes that are both organ specific and lineage-specific had been proposed [11]. In bacteria, lineagespecificity reference genes referred to suitable reference genes across different species, such as across genus (known as genus-specific reference genes) or across different families (known as family-specific reference genes). Hence, reference genes both lineage-specific and organ specific (known as lineage-organ specific reference genes) referred to suitable reference genes for a particular organ across different species. For example, a suitable spleen reference gene suitable for all species of mammals would be known as mammal-spleenspecific reference gene. However, if the reference gene was only suitable for marsupial spleen, then it would be known as a marsupial-spleen-specific reference gene.

In this study, we evaluated 2 liver transcriptome microarray datasets from $S$. lateralis and $S$. tridecemlineatus to draw conclusions as to whether there are reference genes that are both genus specific and liver specific. The liver transcriptomes of Spermophilus lateralis, a ground-roaming golden-mantle ground squirrel, and Spermophilus tridecemlineatus, a treehabiting 13-lined ground squirrel, under different states of feeding and hibernation had been studied using the same microarray platform (GPL 1706; [26]). This allows for transcriptome comparison between two closely related species as the experimental conditions were largely similar.

\section{Materials and Methods}

2.1. Microarray Data. Two datasets were obtained from publicly available microarray database, Gene Expression Omnibus, National Centre for Biotechnology Information, of which one of them was from S. lateralis consisting of 35 samples (GSE2024) and another was from S. tridecemlineatus consisting of 26 samples (GSE2021). Briefly, these gene expression datasets represent the liver transcriptome for animals sampled during summer, interbout arousal, and late torpor [26].

2.2. Normalization across Data Sets. The intensity of each probe was calculated as the log-ratio of liver reference against sample. After which, each original probe log-ratio value in the original dataset was normalized using $Z$-score transformation based on the method previously described $[11,27]$. Briefly, $Z$-score Probe $=\left(\right.$ Probe $_{\text {Initial }} \times\left(\mu_{\text {InitialProbe }}\right)$ $\left.\left.\mu_{\text {Assumed }}\right)-\mu_{\text {Assumed }}\right) / \mathrm{SD}_{\text {Dataset }}$, where Probe Initial $_{\text {is the origi- }}$ nal probe log-ratio intensity value in the dataset, $\mu_{\text {InitialProbe }}$ is the mean of the initial probe log-ratio values for a particular

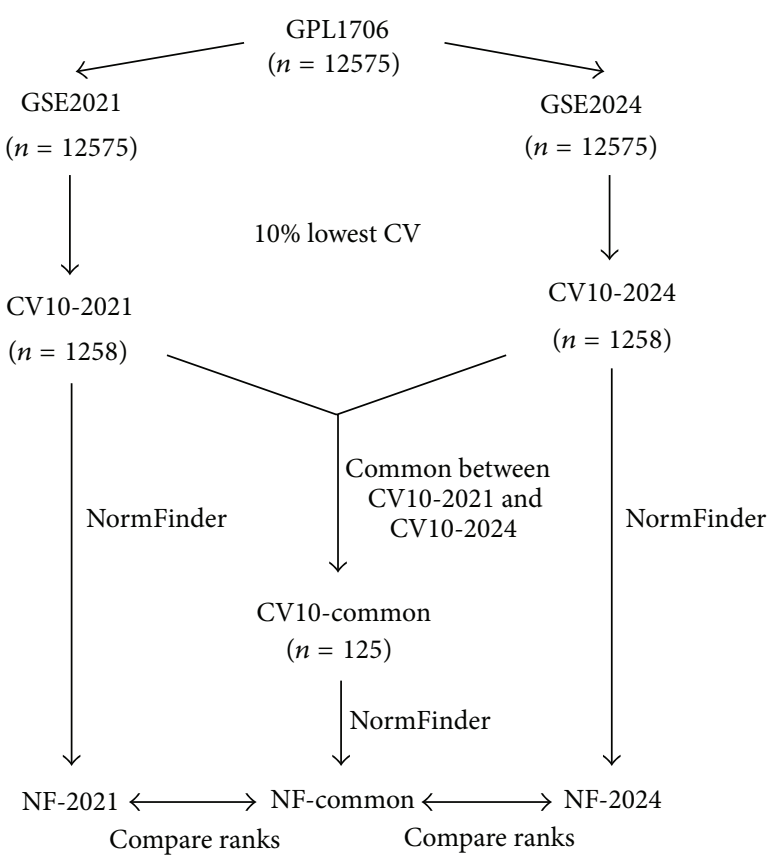

Figure 1: Summary of materials and methods. Two datasets, GSE2021 and GSE2024, were obtained from NCBI GEO, and probes were ranked by coefficient of variation $(\mathrm{CV})$. Probes with the lowest decile in terms of CV were identified from each dataset as CV102021 and CV10-2014, respectively. The set of probes found in both CV10-2021 and CV10-2014 was identified as CV10-common. All 3 sets: CV10-2021, CV10-2014, and CV10-common, were analyzed by NormFinder [6].

sample, $\mu_{\text {Assumed }}$ is mean of both datasets consisting of 61 samples, and $\mathrm{SD}_{\text {Dataset }}$ is the standard deviation for each initial dataset. The $Z$-scores for each probe across different original datasets will then be comparable. The absolute values for $Z$-scores were used for further analyses.

2.3. Determining Correlation between Datasets. The coefficient of variation (CV) of every probe was calculated as the quotient of standard deviation and arithmetic mean. From 12575 probes, the probes were ranked in ascending $\mathrm{CV}$ for each dataset, and the rank difference was calculated. Spearman's correlation was used to determine the correlation between the CV values between GSE2021 and GSE2024. From 12575 probes, the data was separated into groups of $10 \%$ each. The top $10 \%$ of probes in GSE2021 and GSE2024 were identified. These were called CV10-2021 and CV102024, respectively (Figure 1). A third list, CV10-common, was defined as common probes between CV10-2021 and CV102024. All three lists were analysed using NormFinder version 0.953 [6] to rank the stability of these probes separately by estimating the expressional variation of the probe with respect to the overall variation in the entire dataset. The resulting NormFinder outputs from CV10-2021, CV10-2024, and CV10-common were referred to as NF-2021, NF-2024, and NF-common, respectively. Spearman's correlation of the NormFinder stability index values between all sets was calculated and evaluated for the null hypothesis of no correlation 
between the data sets using $Z$-test for correlation coefficient $[8,28]$.

2.4. Bootstrap Statistics. The significance of overlap, CV10common, was determined using bootstrapping method [29] where the percentage of overlap is the test statistic. The bootstrap distribution was generated by the percentage overlap of 2000 repetitive resampling from the original data with replacement to yield samples of the same sizes as that used to calculate test statistic (see Appendix A). Z-test was performed on the test statistic using the mean and standard deviation of the bootstrap distribution.

2.5. Expression Correlation of Invariant Probes. The expression correlations of the invariant probes with other probes in GSE2021 were found. This tests the hypothesis that the expressions of invariant probes were less correlated to the transcriptome compared to randomly selected probes. Ten least variant probes (10 most expressionally stable probes) were identified from NF-2021 and denoted as NF-10. Ten probes which were not in NF-10 were randomly selected from GSE2021 ( $n=12565)$ and denoted as Random-10. The rest of the 12,555 probes that were not in NF-10 or Random-10 were denoted as Others-2021. Pair-wise Pearson's correlation is performed between each of the probes in NF-10 and each of the probes in Others-2021 to give a sample of the expression correlation of invariant probes and other probes (total correlations $=125,550)$. The random correlations were estimated by pair-wise correlation between each probe in Random-10 and Others-2021. To prevent biasness, the process of randomly selecting 10 probes that were not in NF10 and pair-wise correlation calculation was repeated 5 times.

\section{Results}

The two datasets used in this study, GSE2021 and GSE2024, were of the same microarray platform (GPL1706), examining differential gene expressions in the liver of 2 species of squirrels in similar states of hibernation and activity. This allows for a comparative study to evaluate potential genusspecific and organ-specific reference genes. The microarray consists of 12575 probes. Spearman's correlation coefficient of the coefficient of variation (CV) of the probes in GSE2021 and GSE2024 after $Z$-normalization was $0.141(P$ value $=1 e-56)$, suggesting correlation between both datasets.

Among the lowest $10 \%$ of CV in both datasets (CV102021 and CV10-2024, $n=1258), 178$ probes (14.2\%) were common in both datasets, which is statistically significant from a bootstrap mean of $43.11 \%$ with a bootstrap standard deviation of $1.33 \%$ ( $P$ value $<1 e-300)$ as resampled from the lowest $10 \%$ of $\mathrm{CV}$ in both datasets. However, Spearmans correlation for the NormFinder ranks of these 178 common probes between GSE2021 and GSE2024 was 0.322, and Z-test suggests that the ranks are correlated $(P$ value $=1 e-5)$.

CV10-2021, CV10-2024, and CV10-common were analysed by NormFinder. There is one common probe (Probe ID 07I20, Table 1) between the 20 most invariant probes of NF-2021 and NFC-2021 (S. tridecemlineatus). NFC-2021 is
TABLE 1: Top 20 invariant probes of GSE2021 (S. tridecemlineatus) by NormFinder. NF-2021 is the result of NormFinder analysis of CV10-2021. NFC-2021 is the result of NormFinder analysis of CV10common, using data from GSE2021. There is one common probe between NF-2024 and NFC-2024 as marked by asterisks.

\begin{tabular}{lccc}
\hline \multicolumn{2}{c}{ NFC-2021 } & \multicolumn{2}{c}{ NF-2021 } \\
Probe & Stability index & Probe & Stability index \\
\hline $12 \mathrm{~g} 21$ & 0.230 & $02 \mathrm{n} 12$ & 0.167 \\
07120 & $0.230\left(^{*}\right)$ & $13 \mathrm{~d} 19$ & 0.195 \\
$01 \mathrm{~h} 05$ & 0.245 & $05 \mathrm{p} 15$ & 0.197 \\
$12 \mathrm{c} 07$ & 0.247 & $02 \mathrm{~g} 16$ & 0.201 \\
$01 \mathrm{~g} 24$ & 0.259 & $03 \mathrm{o} 23$ & 0.206 \\
$15 \mathrm{~d} 12$ & 0.267 & $03 \mathrm{j} 13$ & 0.208 \\
$12 \mathrm{c} 08$ & 0.272 & $13 \mathrm{~d} 08$ & 0.208 \\
$01 \mathrm{k} 05$ & 0.281 & $28 \mathrm{~h} 07$ & 0.219 \\
$12 \mathrm{~h} 15$ & 0.282 & $13 \mathrm{o} 12$ & 0.220 \\
$01 \mathrm{o} 21$ & 0.282 & $12 \mathrm{p} 20$ & 0.221 \\
$12 \mathrm{o} 20$ & 0.291 & $28 \mathrm{~h} 10$ & 0.225 \\
$23 \mathrm{k} 15$ & 0.291 & $28 \mathrm{n} 23$ & 0.229 \\
$15 \mathrm{k} 15$ & 0.292 & $26 \mathrm{~h} 02$ & 0.233 \\
$12 \mathrm{~d} 19$ & 0.295 & $13 \mathrm{~b} 20$ & 0.237 \\
$12 \mathrm{k} 13$ & 0.296 & 07120 & $0.243\left(^{*}\right)$ \\
$23 \mathrm{p} 12$ & 0.299 & $15 \mathrm{~b} 12$ & 0.246 \\
23115 & 0.319 & $13 \mathrm{c} 20$ & 0.249 \\
$26 \mathrm{~h} 04$ & 0.322 & $01 \mathrm{k} 09$ & 0.251 \\
$12 \mathrm{~g} 19$ & 0.323 & $03 \mathrm{o} 04$ & 0.251 \\
$28 \mathrm{~h} 04$ & 0.328 & $12 \mathrm{o} 11$ & 0.252 \\
\hline
\end{tabular}

the result of NormFinder analysis of CV10-common, using data from GSE2021. Coversely, NFC-2024 is the result of NormFinder analysis of CV10-common, using data from GSE2024. When ranked by NormFinder stability indices, the ranks of Probe ID 07I20 are 926 and 127 in NF-2024 and NFC2024 (S. lateralis), respectively.

However, there are 6 common probes in the 20 most invariant probes of NF-2024 and NFC-2024 (S. lateralis; Table 2), including the top 2 invariant probes, $24 \mathrm{j} 21$ and $12 \mathrm{c} 07$. The NormFinder stability ranks of Probe IDs 24j21, 12c07, $24 \mathrm{n} 06,12 \mathrm{~g} 19,15 \mathrm{~b} 23$, and 12j13 in NF-2021 (S. tridecemlineatus) are $8644,84,321,225,654$, and 650 , respectively. Similarly, the NormFinder stability ranks of Probe IDs 24j21, 12c07, 24n06, $12 \mathrm{~g} 19,15 \mathrm{~b} 23$, and 12j13 in NFC-2021 (S. tridecemlineatus) are $127,4,53,19,101$, and 89 , respectively.

There is no common probe found within all 4 lists, namely, NF-2021, NFC-2021, NF-2024, and NFC-2024.

The average coefficient of determination $\left(r^{2}\right)$ between 10 of the most invariant probes of GSE2021 (NF-10) and Others2021 (those probes not found in NF-10 or Random-10) was 0.055 with standard deviation of 0.136 . The average coefficient of determination $\left(r^{2}\right)$ of the 5 replicates between (Random10) and Others-2021 ranged from 0.115 to 0.147 with standard deviation ranged from 0.133 to 0.167 (Figure 2). Using $t$-test with unequal variance, the $r^{2}$ between NF-10 and Others2021 was significantly lower than the $r^{2}$ between Random2021 and Others-2021 ( $P$ value $<1 e-300)$. By calculating 
TABLE 2: Top 20 invariant probes of GSE2024 (S. lateralis) by NormFinder. NF-2024 was the result of NormFinder analysis of CV10-2024. NFC-2024 was the result of NormFinder analysis of CV10-common, using data from GSE2024. Common probes in NF2024 and NFC-2024 were marked by asterisks.

\begin{tabular}{lccc}
\hline \multicolumn{2}{c}{ NFC-2024 } & \multicolumn{2}{c}{ NF-2024 } \\
Probe & Stability index & Probe & Stability index \\
\hline $24 \mathrm{j} 21$ & $0.269\left(^{*}\right)$ & $24 \mathrm{j} 21$ & $0.359\left(^{*}\right)$ \\
$12 \mathrm{c} 07$ & $0.298\left(^{*}\right)$ & $12 \mathrm{c} 07$ & $0.391\left(^{*}\right)$ \\
$12 \mathrm{~g} 21$ & 0.324 & $27 \mathrm{j} 16$ & 0.406 \\
$15 \mathrm{k} 15$ & 0.328 & $26 \mathrm{~b} 14$ & 0.415 \\
$24 \mathrm{n} 06$ & $0.334\left(^{*}\right)$ & $24 \mathrm{n} 06$ & $0.422\left(^{*}\right)$ \\
$12 \mathrm{~g} 19$ & $0.339\left(^{*}\right)$ & $14 \mathrm{p} 14$ & 0.427 \\
$12 \mathrm{j} 13$ & $0.355\left(^{*}\right)$ & $18 \mathrm{o} 04$ & 0.441 \\
$27 \mathrm{o} 22$ & 0.363 & $12 \mathrm{~g} 19$ & $0.454\left(^{*}\right)$ \\
$15 \mathrm{~b} 23$ & $0.374\left(^{*}\right)$ & $23 \mathrm{n} 15$ & 0.455 \\
$13 \mathrm{k} 06$ & 0.379 & $23 \mathrm{n} 05$ & 0.466 \\
$12 \mathrm{c} 11$ & 0.380 & $23 \mathrm{~h} 06$ & 0.476 \\
$13 \mathrm{k} 07$ & 0.383 & $24 \mathrm{~h} 23$ & 0.477 \\
$13 \mathrm{j} 13$ & 0.383 & 13115 & 0.479 \\
$33 \mathrm{p} 05$ & 0.383 & $02 \mathrm{o} 05$ & 0.480 \\
$33 \mathrm{p} 07$ & 0.393 & $23 \mathrm{n} 04$ & 0.485 \\
$15 \mathrm{~g} 15$ & 0.406 & $26 \mathrm{~g} 06$ & 0.486 \\
$24 \mathrm{n} 07$ & 0.424 & $15 \mathrm{~b} 23$ & $0.488\left(^{*}\right)$ \\
$23 \mathrm{j} 20$ & 0.428 & $27 \mathrm{c} 12$ & 0.490 \\
$33 \mathrm{e} 16$ & 0.431 & $12 \mathrm{f} 02$ & 0.492 \\
$13 \mathrm{~b} 14$ & 0.432 & $12 \mathrm{j} 13$ & $0.503\left(^{*}\right)$ \\
\hline & & &
\end{tabular}

Pearson's correlations of the expression of a probe with itself, the floating-point error in calculation can be estimated to be zero with a standard deviation of $2.79 e-12$. Hence, we reject the null hypothesis that the correlations between invariant probes and random variant probes are equal and accept the alternate hypothesis that they are significantly lower.

\section{Discussion}

Reference genes play a crucial role as standards for normalizing expression values in quantitative gene expression experiments. Common reference genes such as GAPDH [19] and UBQ [18] can be used over a very wide range of organisms, but individual studies suggested that these genes are not optimal and there exist more reliably stable genes within each single species [10,21]. Genes that are suitable as reference genes for one organism may not be suitable for another [25]. However, the possibility of lineage-organspecific reference genes had been proposed [11]. Using 2 microarray datasets from squirrel liver of 2 species under the same genus obtained under comparable conditions [26], this study aims to identify and evaluate potential genus-specific and liver-specific reference genes suitable for use in both $S$. lateralis and S. tridecemlineatus, as well as to draw a general conclusion to the similarity of the general gene expression levels in both species.

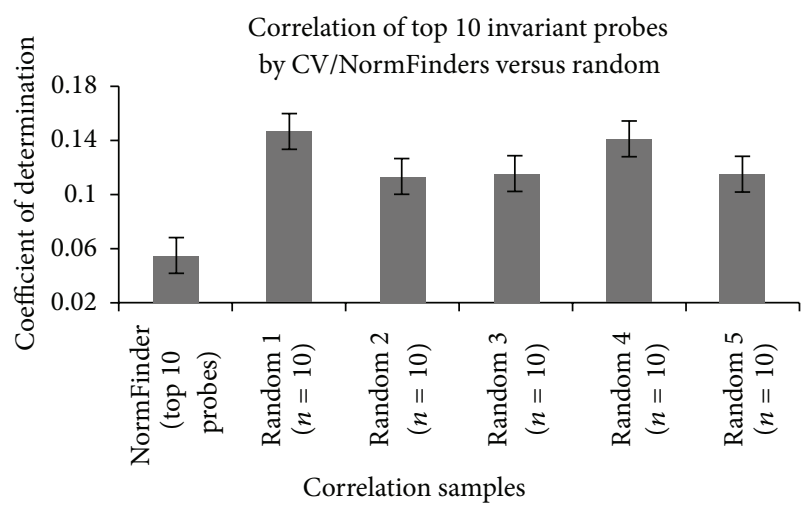

FIGURE 2: Average correlation of Top 10 invariant probes by $\mathrm{CV} /$ NormFinder (NF-10) against random (Random-10). Five replicates of correlations between Random-10 and Others-2021 were performed. Error bars denote standard error.

Spearman's correlation $\left(P\right.$ value $\left.=1.00 \times 10^{-56}\right)$ for the entire dataset $(n=12575)$ based on CV ranks showed correlation between GSE 2021 and GSE 2024, suggesting transcriptional similarities between the livers of $S$. lateralis and S. tridecemlineatus. This supports Williams et al. [26]. Their paper found consistency in gene regulation between squirrel species, relating to different biological classes of genes. Such correlation in our data is expected as the two squirrel species are from the same genus. In addition, the data was collected from the same organ with the same experimental conditions [26]. Thus, the expression profiles are assumed to be largely similar. Moreover, Spearman's correlation for the 178 probes in NF-common also revealed a high correlation $\left(P\right.$ value $\left.=1.10 \times 10^{-5}\right)$. This is also expected, due to the overall correlation of the whole dataset. In addition, Natale et al. [30] found similar gene expressions after brain trauma in mice and rats of different genera. Albert et al. [31] found less than 1\% differences between gene expression of dogs and wolves and other domesticated and wild animals. These results support our hypothesis.

The ratio of overlap between the $10 \%$ lowest $\mathrm{CV}$ of both datasets (CV10-common) is $14.2 \%$, which is much lesser than the expected $43.1 \%$ from bootstrapping. This result is significant $(P$ value $<1 e-300)$, showing that there is little correlation between the invariance of a gene in $S$. lateralis and its invariance in S. tridecemlineatus, which suggests that genus-specific reference genes are unlikely. Moreover, our results demonstrated a lack of commonality between the top 20 probes in NF-2021 and NF-2024. This implies that the suitable liver reference probes identified by NormFinder for S. tridecemlineatus (GSE2021) are not suitable for S. lateralis.

However, there are 6 probes in the top 20 of NF2024 that also fall in NFC-2024 (NF-common of GSE2024), but these probes are ranked poorly in NF2021 (S. tridecemlineatus) with 84 the best rank. This suggests that there are 83 more suitable reference probes for S. tridecemlineatus if Probe $12 \mathrm{c} 07$ is to be used as a reference probe across both species. In addition, our results showed that the most stable probe for $S$. lateralis (Probe 24j21) is ranked 8644 in S. tridecemlineatus. Similarly, 


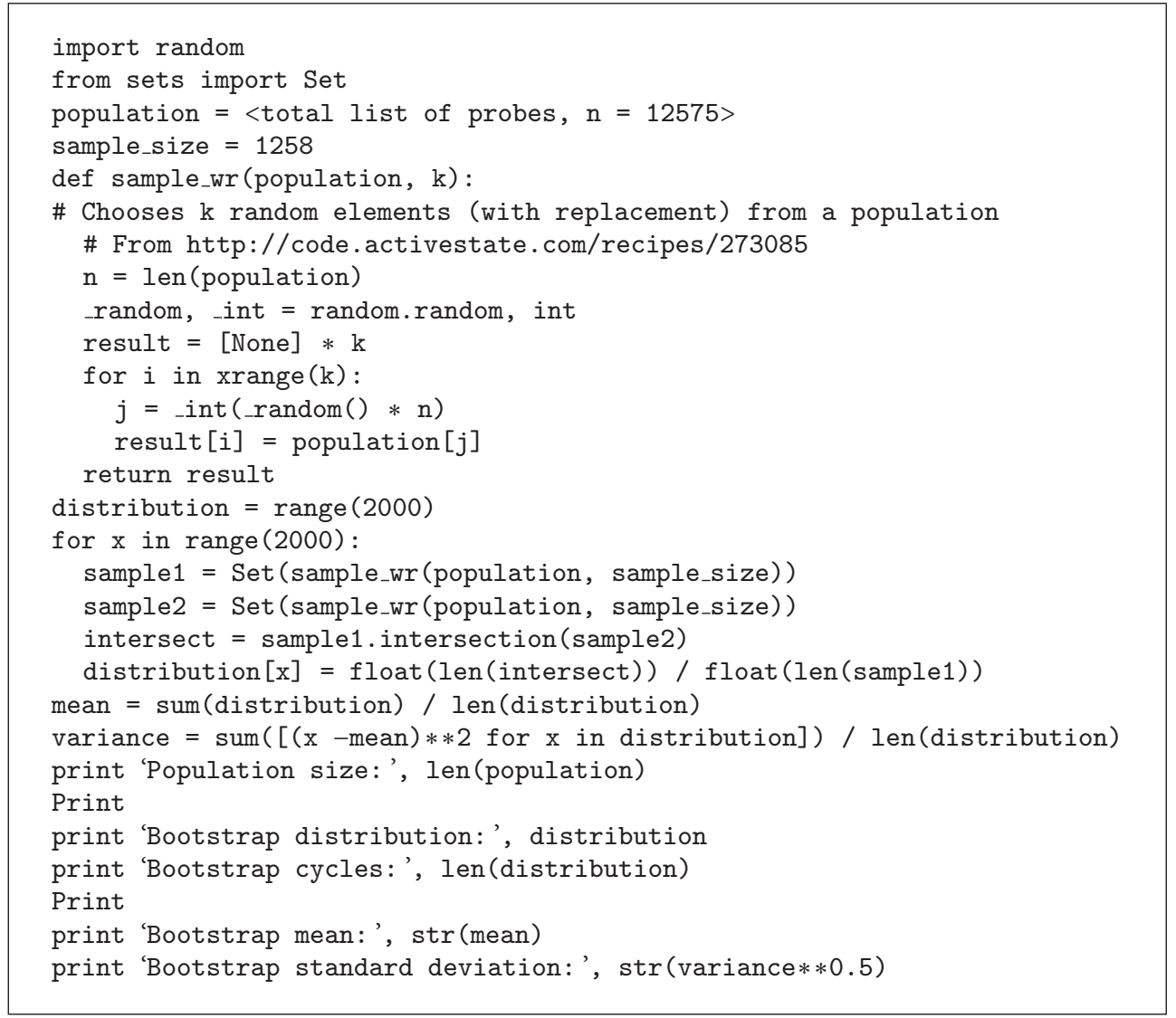

Algorithm 1: Python codes for overlap analysis by bootstrapping.

the common probe for NF-2021 and NFC-2021 (S. tridecemlineatus), Probe 07120, is ranked lowly for S. lateralis (ranked 926 and 127 respectively). This is supported by our findings that there are no common probes in the top 20 NormFinder identified reference probes of NF-2021 (S. tridecemlineatus) and NF-2024 (S. lateralis). A suitable reference probe for $S$. lateralis and $S$. tridecemlineatus, by extension, a genus-liverspecific reference probe for S. lateralis and S. tridecemlineatus, should have low ranks in both datasets. Taken together, these results show that the reference probes suitable for one species may not be suitable for other closely related species, which also suggests that genus-specific reference genes are unlikely.

A plausible explanation for the previous results is gene function degeneracy across different species. It has been suggested that redundant genetic networks may be present to maintain network robustness of cellular function [3234]. This indicates that not all genes may be critical because certain functional overlaps allow interchanging of functions due to functional degeneracy. Furthermore, it has also been suggested that cells have some buffer in genetic expressions [35-37]. Thus, when expression of a particular gene is altered, such a buffering mechanism enables different genes to fulfil a similar function. This is a possible explanation why the transcriptome of invariant genes between the $S$. lateralis and $S$. tridecemlineatus has a poor overlap for the genes of lowest invariance while there is significant correlation for the datasets as a whole, indicating that a particular function is not performed by the same genes in both species. Yet a previously conducted study [26] has stated that the overall liver functions of the 2 squirrels are similar. Therefore, this corroborates the hypothesis that different genes can serve a similar function.

There is discrepancy in the overlap of the sets of differentially expressed genes in the two hibernating species, implying that the overlaps are not as significant as expected. In other words, the genes are not closely related. This may be attributed to difficulties in the statistical detection of the modest transcriptional changes between the summer and winter animals, changes that may be masked due to interindividual variation and the difference in the number of individuals analyzed for each species (26 samples in GSE2021 and 35 samples in GSE2024) [26]. However, interindividual variation and different numbers of animals studied by Williams et al. [26] are unlikely to be substantial enough to result in a lack of suitable reference probes between 2 closely related species of squirrels. A possible explanation to reconcile the observation that overall liver functions of the 2 squirrels is similar [26] and yet demonstrating the lack of suitable common reference probes may be a situation whereby the topology of the gene expressions between the 2 species are similar but individual gene expressions may vary. Moreno-Sánchez et al. [38] and Ling et al. [28] had 


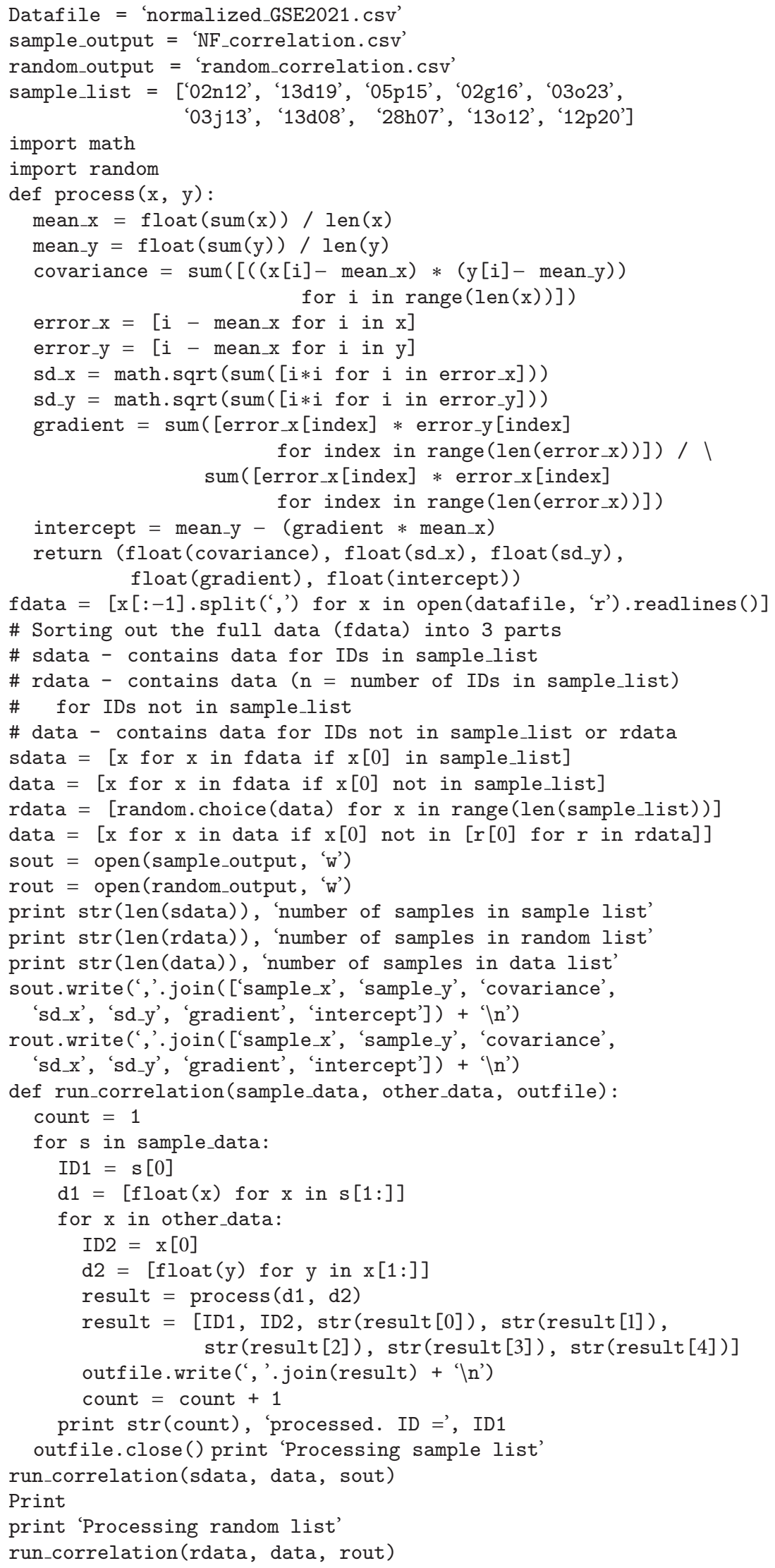

Algorithm 2: Python codes for expression analysis. 
demonstrated that if 2 genes are expressionally correlated, there is a strong chance that these 2 genes are functionally related. Further studies examining the coexpression networks from these 2 datasets may be able to elucidate the impact of the expressional variation of individual genes on the topology of expression.

Our results demonstrate that the correlation between the NF-10 probes (invariant) and the Others-2021 (variant) is low $\left(r^{2}=0.055\right)$, whereas the coefficient of determination $\left(r^{2}\right)$ between the randomly selected probes (Random-10) and probes from Others-2021 ranges from 0.115-0.147. This result is significant $(P$ value $<1 e-300)$, rejecting the null hypothesis that correlation between invariant probes (NFprobes) is equal to correlation between variant (random) probes. This supports the use of correlation as a method to identify reference probes/genes, which had been previously reported [39].

The data used in this study is from these two closely related species under the same experimental conditions and from the same organ. However, our results suggests that reference probes found in one species may not be suitable in other as we are not able to conclusively determine suitable genus-specific reference genes even in such closely related species and for the same organ. Despite having similar organ transcriptomes [26], our results do not support the presence of highly stable genus-organ-specific reference genes or probes even though we found several candidates of potential reference probes for both species using a lowest denominator approach. However, this also suggests that the suitability of potential reference genes or probes across more diverse species will be questionable. Therefore, it can be extrapolated that reference genes that are stable over an even wider range of organisms across genus and families would be unlikely to exist. This shows that for accurate experimentation, new reference genes should be identified for each organism and for each organ individually.

\section{Conclusion}

In conclusion, our results do not suggest the presence of a liver-specific reference gene/probe by analysing transcriptome profiles of 2 closely related species under similar conditions despite evidence supporting previous studies on the similarity of the transcriptomes. Hence, this study does not support the presence of genus-organ-specific reference gene. Therefore, reference genes should be identified for each organism and for each organ individually.

\section{Appendices}

\section{A. Python Codes for Overlap Analysis by Bootstrapping}

For more details, see Algorithm 1.

\section{B. Python Codes for Expression Analysis}

For more details, see Algorithm 2.

\section{Authors' Contribution}

Bryan M. H. Keng, Oliver Y. W. Chan, and Sean S. J. Heng contributed equally to this work.

\section{Acknowledgments}

The authors wish to thank ECK Wong (The University of Melbourne) and YZ Koh (University of Portsmouth) for their comments.

\section{References}

[1] O. Fedrigo, L. R. Warner, A. D. Pfefferle, C. C. Babbitt, P. CruzGordillo, and G. A. Wray, "A pipeline to determine RT-QPCR control genes for evolutionary studies: application to primate gene expression across multiple tissues," PLOS ONE, vol. 5, no. 9, Article ID e12545, pp. 1-7, 2010.

[2] T. Remans, K. Smeets, K. Opdenakker, D. Mathijsen, J. Vangronsveld, and A. Cuypers, "Normalisation of real-time RTPCR gene expression measurements in Arabidopsis thaliana exposed to increased metal concentrations," Planta, vol. 227, no. 6, pp. 1343-1349, 2008.

[3] N. Agabian, L. Thomashow, M. Milhausen, and K. Stuart, "Structural analysis of variant and invariant genes in trypanosomes," American Journal of Tropical Medicine and Hygiene, vol. 29, no. 5, pp. 1043-1049, 1980.

[4] D. T. Le, D. L. Aldrich, B. Valliyodan et al., "Evaluation of candidate reference genes for normalization of quantitative RTPCR in soybean tissues under various abiotic stress conditions," PloS One, vol. 7, no. 9, Article ID e46487, 2012.

[5] J. Vandesompele, K. De Preter, F. Pattyn et al., "Accurate normalization of real-time quantitative RT-PCR data by geometric averaging of multiple internal control genes," Genome biology, vol. 3, no. 7, 2002.

[6] C. L. Andersen, J. L. Jensen, and T. F. Ørntoft, "Normalization of real-time quantitative reverse transcription-PCR data: a modelbased variance estimation approach to identify genes suited for normalization, applied to bladder and colon cancer data sets," Cancer Research, vol. 64, no. 15, pp. 5245-5250, 2004.

[7] M. Kubista, R. Sindelka, A. Tichopad, A. Bergkvist, D. Lindh, and A. Forooran, "The prime technique. Real-time PCR data analysis," G.I.T. Laboratory Journal, vol. 9, no. 10, pp. 33-35, 2007.

[8] G. Kanji, 100 Statistical Tests, Sage, 3rd edition, 2006.

[9] E. Wurmbach, T. Yuen, and S. C. Sealfon, "Focused microarray analysis," Methods, vol. 31, no. 4, pp. 306-316, 2003.

[10] S. S. J. Heng, O. Y. W. Chan, B. M. H. Keng, and M. H. T. Ling, "Glucan biosynthesis protein G, (mdoG) is a suitable reference gene in Escherichia coli K-12," ISRN Microbiology, vol. 2011, Article ID 469053, 6 pages, 2011.

[11] I. H. K. Too and M. H. T. Ling, "Signal peptidase complex subunit 1 and hydroxyacyl-CoA dehydrogenase beta subunit are suitable reference genes in human lungs," ISRN Bioinformatics, vol. 2012, Article ID 790452, 7 pages, 2012.

[12] T. Czechowski, M. Stitt, T. Altmann, M. K. Udvardi, and W. R. Scheible, "Genome-wide identification and testing of superior reference genes for transcript normalization in arabidopsis," Plant Physiology, vol. 139, no. 1, pp. 5-17, 2005.

[13] M. Jain, A. Nijhawan, A. K. Tyagi, and J. P. Khurana, "Validation of housekeeping genes as internal control for studying gene 
expression in rice by quantitative real-time PCR," Biochemical and Biophysical Research Communications, vol. 345, no. 2, pp. 646-651, 2006.

[14] N. Nicot, J. F. Hausman, L. Hoffmann, and D. Evers, "Housekeeping gene selection for real-time RT-PCR normalization in potato during biotic and abiotic stress," Journal of Experimental Botany, vol. 56, no. 421, pp. 2907-2914, 2005.

[15] U. E. M. Gibson, C. A. Heid, and P. M. Williams, "A novel method for real time quantitative RT-PCR," Genome Research, vol. 6, no. 10, pp. 995-1001, 1996.

[16] S. R. Sturzenbaum and P. Kille, "Control genes in quantitative molecular biological techniques: the variability of invariance," Comparative Biochemistry and Physiology B, vol. 130, pp. 281289, 2001.

[17] G. W. Takle, I. K. Toth, and M. B. Brurberg, "Evaluation of reference genes for real-time RT-PCR expression studies in the plant pathogen Pectobacterium atrosepticum," BMC Plant Biology, vol. 7, article 50, 2007.

[18] N. C. Noriega, S. G. Kohama, and H. F. Urbanski, “ $\kappa$ Microarray analysis of relative gene expression stability for selection of internal reference genes in the rhesus macaque brain," $B M C$ Molecular Biology, vol. 11, article 47, 2010.

[19] E. M. Glare, M. Divjak, M. J. Bailey, and E. H. Walters, “ $\beta$ actin and GAPDH housekeeping gene expression in asthmatic airways is variable and not suitable for normalising mRNA levels," Thorax, vol. 57, no. 9, pp. 765-770, 2002.

[20] L. Gutierrez, M. Mauriat, S. Guénin et al., “The lack of a systematic validation of reference genes: a serious pitfall undervalued in reverse transcription-polymerase chain reaction (RT-PCR) analysis in plants," Plant Biotechnology Journal, vol. 6, no. 6, pp. 609-618, 2008.

[21] T. Brattelid, L. H. Winer, F. O. Levy, K. Liestøl, O. M. Sejersted, and K. B. Andersson, "Reference gene alternatives to Gapdh in rodent and human heart failure gene expression studies," $B M C$ Molecular Biology, vol. 11, article 22, 2010.

[22] S. Mamo, A. B. Gal, S. Bodo, and A. Dinnyes, "Quantitative evaluation and selection of reference genes in mouse oocytes and embryos cultured in vivo and in vitro," BMC Developmental Biology, vol. 7, article 14, 2007.

[23] S. Pérez, L. J. Royo, A. Astudillo et al., "Identifying the most suitable endogenous control for determining gene expression in hearts from organ donors," BMC Molecular Biology, vol. 8, article 114, 2007.

[24] C. Pfister, M. S. Tatagiba, and F. Roser, "Selection of suitable reference genes for quantitative real-time polymerase chain reaction in human meningiomas and arachnoidea," BMC Research Notes, vol. 4, article 275, 2011.

[25] J. B. Dundas and M. H. T. Ling, "Reference genes for Measuring mRNA Expression," Theory in Biosciences, vol. 131, pp. 215-223, 2012.

[26] D. R. Williams, L. E. Epperson, W. Li et al., "Seasonally hibernating phenotype assessed through transcript screening," Physiological Genomics, vol. 24, no. 1, pp. 13-22, 2005.

[27] C. Cheadle, Y. S. Cho-Chung, K. G. Becker, and M. P. Vawter, "Application of z-score transformation to Affymetrix data," Applied Bioinformatics, vol. 2, no. 4, pp. 209-217, 2003.

[28] M. H. T. Ling, C. Lefevre, and K. R. Nicholas, "Filtering microarray correlations by statistical literature analysis yields potential hypotheses for lactation research," The Python Papers, vol. 3 , no. 3, article 4, 2008 .

[29] B. Efron and R. Tibshirani, An Introduction to the Bootstrap, Chapman \& Hall/CRC, Boca Raton, Fla, USA, 1993.
[30] J. E. Natale, F. Ahmed, I. Cernak, B. Stoica, and A. I. Faden, "Gene expression profile changes are commonly modulated across models and species after traumatic brain injury," Journal of Neurotrauma, vol. 20, no. 10, pp. 907-927, 2003.

[31] F. W. Albert, M. Somel, M. Carneiro et al., "A comparison of brain gene expression levels in domesticated and wild animals," PLoS Genetics, vol. 8, no. 9, Article ID e1002962, 2012.

[32] J. Li, Z. Yuan, and Z. Zhang, "The cellular robustness by genetic redundancy in budding yeast," PLoS Genetics, vol. 6, no. 11, Article ID e1001187, 2010.

[33] J. Stelling, U. Sauer, Z. Szallasi, F. J. Doyle III, and J. Doyle, "Robustness of cellular functions," Cell, vol. 118, no. 6, pp. 675685, 2004.

[34] G. Thibault, N. Ismail, and D. T. Ng, "The unfolded protein response supports cellular robustness as a broad-spectrum compensatory pathway," Proceedings of the National Academy of Sciences of the United States of America, vol. 108, no. 51, pp. 20597-20602, 2011.

[35] J. M. Whitacre and A. Bender, "Networked buffering: a basic mechanism for distributed robustness in complex adaptive systems," Theoretical Biology and Medical Modelling, vol. 7, no. 1, article 20, 2010.

[36] J. Gómez-Gardeñes, Y. Moreno, and L. M. Floría, "On the robustness of complex heterogeneous gene expression networks," Biophysical Chemistry, vol. 115, no. 2-3, pp. 225-228, 2005.

[37] M. Bekaert and G. C. Conant, "Transcriptional robustness and protein interactions are associated in yeast," BMC Systems Biology, vol. 5, article 62, 2011.

[38] N. Moreno-Sánchez, J. Rueda, M. J. Carabaño et al., "Skeletal muscle specific genes networks in cattle," Functional and Integrative Genomics, vol. 10, no. 4, pp. 609-618, 2010.

[39] S. Lee, M. Jo, J. Lee, S. K. Sang, and S. Kim, "Identification of novel universal housekeeping genes by statistical analysis of microarray data," Journal of Biochemistry and Molecular Biology, vol. 40, no. 2, pp. 226-231, 2007. 

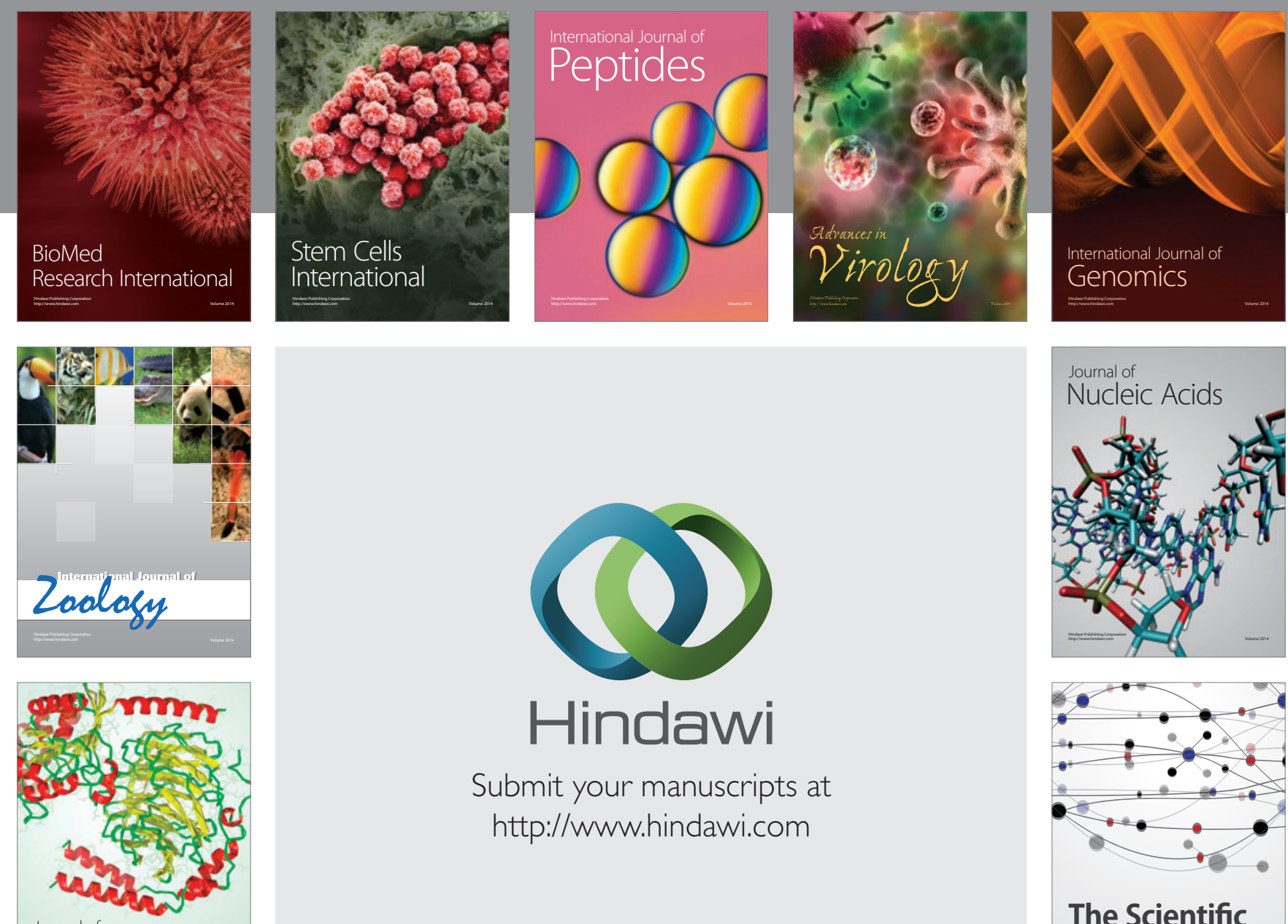

Submit your manuscripts at

http://www.hindawi.com

Journal of
Signal Transduction
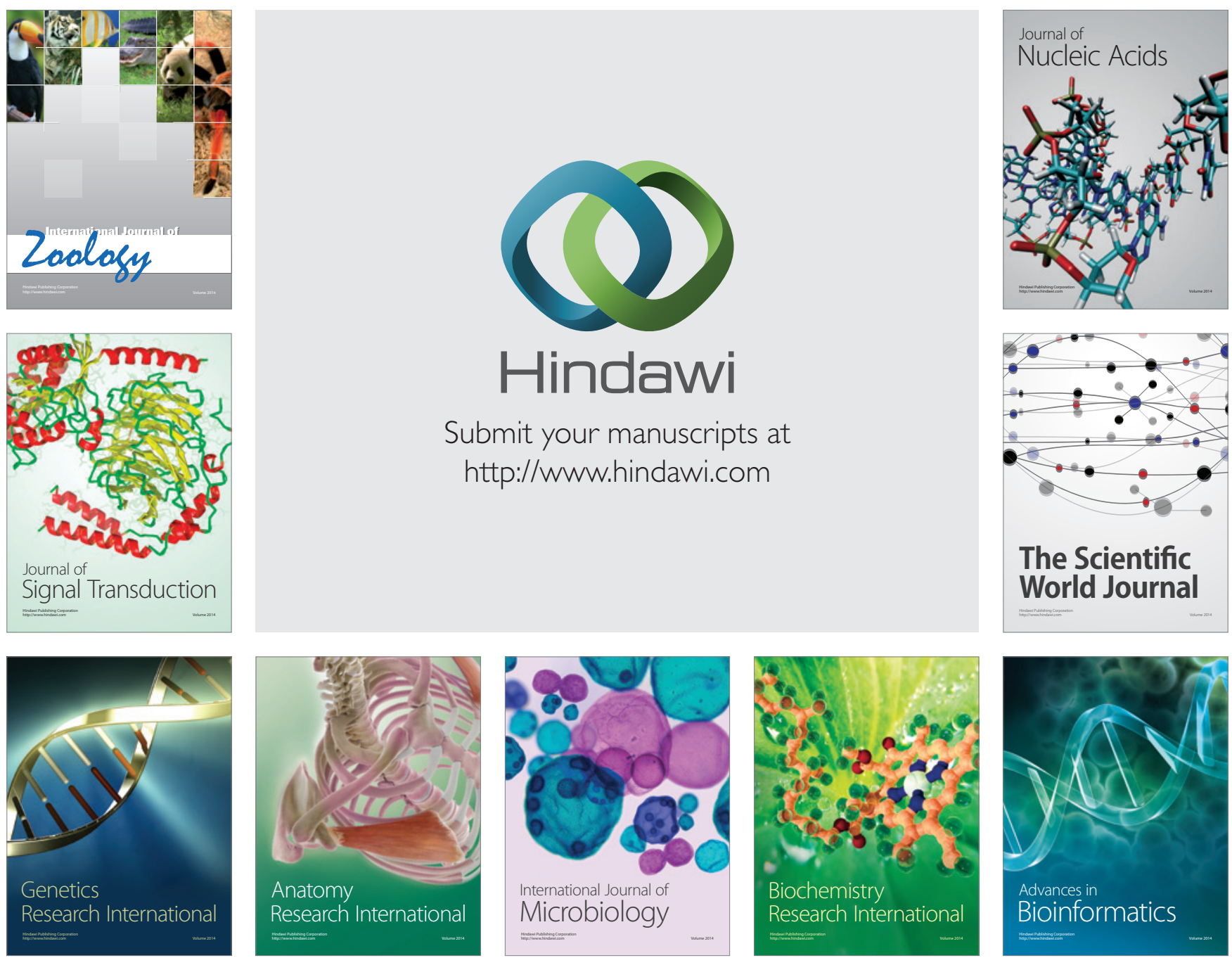

The Scientific World Journal
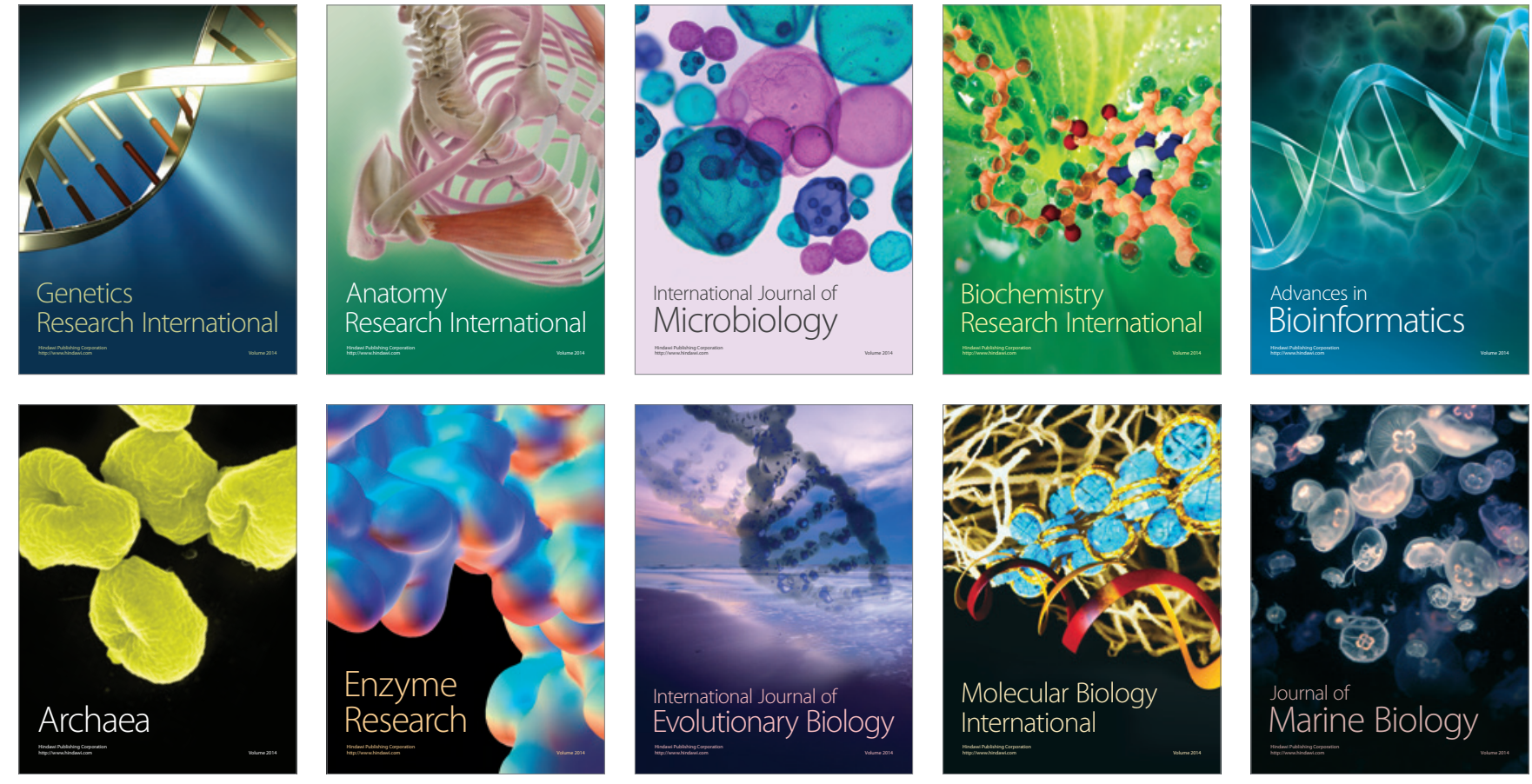